\title{
ANÁliSE DE TRILHA ENTRE AS VARIÁVEIS DAS ANÁLISES DE SEMENTES DE ESPÉCIES FLORESTAIS EXÓTICAS DO RIO GRANDE DO SUL ${ }^{1}$
}

\author{
Leandro Homrich Lorentz ${ }^{2}$, Fabiano de Oliveira Fortes ${ }^{3}$ e Alessandro Dal'col Lúcio ${ }^{4}$
}

\begin{abstract}
RESUMO - Este trabalho teve por objetivo identificar as variáveis mais importantes na avaliação da qualidade das sementes armazenadas de espécies florestais exóticas no sul do Brasil. As informações relacionadas às análises de sementes das espécies florestais exóticas foram obtidas no banco de dados do Centro de Pesquisas Florestais e Conservação do Solo - FEPAGRO, Santa Maria, RS. Estimaram-se os coeficientes de trilha com as variáveis: pureza dos lotes, peso de mil sementes e porcentagens de umidade, de plântulas normais, de plântulas anormais e de sementes duras e mortas. A porcentagem de sementes mortas e a umidade das sementes armazenadas são as determinações que mais influíram na porcentagem de plântulas normais das espécies florestais exóticas no Rio Grande do Sul.
\end{abstract}

Palavras-chave: Coeficientes de trilha, armazenamento de sementes e controle de qualidade.

\section{PATH ANALYSIS IN SEED ANALYSES OF EXOTIC FOREST SPECIES OF RIO GRANDE DO SUL}

\begin{abstract}
The objective of this study was to identify the most important variables in the evaluation of stored seed quality of exotic forest species of Rio Grande do Sul. Information on seed analyses was obtained from the database of the Forest Research and Soil Conservation Center - FEPAGRO, Santa Maria/RS. Path coefficients were estimated using the variables: lot purity, thousand seed weight, moisture content, percentage of normal and abnormal seedlings, percentage of hard and dead seeds. The percentage of dead seeds and seed moisture are the variables that most influenced the percentage of normal seedlings of exotic forest species of Rio Grande do Sul.
\end{abstract}

Keywords: path coefficients; seed storage, quality control.

\section{INTRODUÇÃO}

Produzir sementes de alta qualidade requer conhecimento e tecnologia. A porcentagem de plântulas normais obtidas nos testes de germinação representa o máximo que a amostra pode oferecer (MACHADO, 2002), uma vez que o teste é conduzido em condições ideais, de forma artificial e obedecendo ao padrão para cada espécie avaliada.

A germinação de sementes, em testes de laboratório, é a emergência das estruturas essenciais do embrião, demonstrando sua aptidão para produzir planta normal sob condições favoráveis de campo, conforme definição em Brasil (1992). A dificuldade de se avaliar qualquer componente da qualidade de um lote de sementes está intimamente relacionada aos problemas da amostragem e ao número de repetições, entre outros (VIEIRA e CARVALHO, 1994).

Coletar dados de forma eficiente, preferivelmente por experimentos planejados, e analisar dados corretamente sendo depois comunicados os resultados

\footnotetext{
${ }^{1}$ Recebido em 22.06.2005 e aceito para publicação em 05.04.2006.

${ }^{2}$ Programa de Pós-Graduação em Agronomia da Universidade Federal de Santa Maria-UFSM97105-900.E-mail:<llorentz@smail.ufsm.br>.

${ }^{3}$ Programa de Pós-Graduação em Engenharia Florestal - UFSM 97105-900. E-mail: <fabfortes@ yahoo.com.br>.

${ }^{4}$ Departamento de Fitotecnia da UFSM 97105-900. E-mail: <adlucio@ smail.ufsm.br. Autor para correspondência>.
} 
aos usuários, para que decisões corretas sejam tomadas, é a melhor forma de obter boas decisões sobre qualidade (GUEDES, 2002). Esse mesmo autor ainda descreveu que o objetivo principal no controle estatístico do processo é reduzir cada vez mais a variabilidade, evitandose, assim, conclusões e inferências equivocadas em relação aos efeitos dos tratamentos avaliados nos diferentes experimentos.

Uma vez que a variabilidade entre as repetições de um tratamento, ou erro experimental, é alta, maiores serão as probabilidades de o pesquisador cometer o erro tipo II, ou seja, não rejeitar a hipótese nula quando esta é falsa. Além dessa consequiência, há um incremento na estatística utilizada para discriminação entre as médias dos tratamentos, fazendo que pequenas diferenças, mesmo sendo significativas, não sejam consideradas como tal (STORCK et al., 2000).

De acordo com as Regras para Análise de Sementes - RAS (BRASIL, 1992), a germinação das sementes é classificada como: a) plântulas normais são aquelas que mostram potencial para continuar seu desenvolvimento e dar origem a plantas normais; b) plântulas anormais são aquelas que não mostram potencial para continuar seu desenvolvimento e dar origem a plântulas normais; c) sementes duras são as que permanecem sem absorver água por um período mais longo que o normal e se apresentam, no final do teste, com aspecto de sementes recém-colocadas no substrato; d) sementes dormentes são as que, embora aparentemente viáveis, não germinam mesmo quando colocadas nas condições especificadas para a espécie em teste; e) sementes mortas são as que, ao final do teste, não germinaram, não estão duras, nem dormentes, e geralmente se mostram amolecidas ou atacadas por microrganismos. Além dessas variáveis, a porcentagem de pureza, o peso de mil sementes e a porcentagem de umidade são avaliados em análise de sementes de espécies florestais e possuem grande variabilidade nas respostas obtidas.

Ao realizar as avaliações de lotes de sementes, há a necessidade de conhecer a interferência do tempo e condições de armazenamento e da espécie trabalhada, sobre a qualidade das sementes armazenadas e no seu potencial de produção de mudas.

O germoplasma é o elemento dos recursos genéticos que maneja a variabilidade genética entre e dentro das espécies (GOEDERT et al., 2002), com fins de utilização para a pesquisa, visando ao melhoramento genético, inclusive a biotecnologia. No entanto, os produtores compram sementes ou mudas de espécies florestais sem ter a garantia da qualidade do lote que estão comprando. Assim, torna-se imprescindível conhecer as características de espécies florestais exóticas cultivadas e comercializadas no Rio Grande do Sul.

O estudo das correlações entre caracteres tem aplicações em praticamente todos os campos de pesquisa. A correlação simples permite apenas avaliar a magnitude e o sentido da associação entre dois caracteres, sem fornecer informações necessárias relativas aos efeitos diretos e indiretos de um grupo de caracteres em relação a uma variável dependente de maior importância (CRUZ, 2001). A análise de trilha permite o estudo dos efeitos de várias variáveis independentes sobre uma variável básica, cujas estimativas são obtidas por meio de equações de regressão em que as variáveis são primeiramente padronizadas, conforme apresentado por Vencovsky e Barriga (1992) e Cruz (2001).

Esse tipo de análise é útil na verificação de relações diretas e indiretas entre variáveis, podendo seu uso ser extrapolado para outras áreas de investigação, além do melhoramento genético, indicando o tipo e o grau de relação entre variáveis e fornecendo condições de melhor planejamento e condução do experimento, podendo também ser aplicada como método de identificação das variáveis menos explicativas do comportamento da variável dependente principal e, assim, eliminá-la do estudo, como descrito por Lúcio (1999).

Dessa forma, este trabalho teve por objetivos identificar as variáveis que mais merecem atenção na avaliação da qualidade das sementes armazenadas de espécies florestais exóticas no Estado do Rio Grande do Sul e verificar suas relações com a variável dependente principal porcentagem de plântulas normais.

\section{MATERIAL E MÉTODOS}

A Fundação Estadual de Pesquisa Agropecuária (FEPAGRO), no Centro de Pesquisas Florestais e Conservação do Solo, em Santa Maria, RS, dispõe de um banco de dados relativo à germinação de sementes florestais exóticas. Desse banco, coletaram-se informações 
sobre a qualidade dos lotes de sementes florestais de diversas espécies exóticas, armazenadas no Banco de Sementes da FEPAGRO, a partir dos laudos correspondentes aos testes de germinação realizados entre janeiro de 1997 e fevereiro de 2003.

As espécies exóticas estudadas foram: acácia (Acacia caven (Mol.) Mol.), calistemun (Calistemon specious DC.), cássia-imperial (Cassia fistula L.), eucalipto (Eucalyptus grandis Hill ex Maiden, Eucalyptus robusta Swamp Mahogany), flamboyant (Delonix regia (Boj.) Rafin), jacarandá-mimoso (Jacaranda mimosaefolia D. Don) e pinus (Pinus elliottii Engl.), que são aquelas presentes no referido banco de sementes (Tabela 1), armazenadas em sacos de papel, a uma temperatura entre 12 e $14^{\circ} \mathrm{C}$ e umidade entre 10 e $15 \%$, sem nenhum tratamento prévio na formação e manutenção dos lotes em armazenamento.

As variáveis utilizadas no estudo foram as avaliadas nas análises de sementes de espécies florestais, que seguem as recomendações de Brasil (1992): peso de mil sementes (PMS) em gramas (para as espécies de eucalipto, o peso de mil sementes é obtido de uma amostra de $0,10 \mathrm{~g}$ ), porcentagens de pureza das sementes (PUR), de umidade (U\%), de plântulas normais (PPN) e plântulas anormais (PPA), de sementes duras (PSD) e de sementes mortas (PSM).

Tabela 1 - Ano de coleta, procedência e número de análises realizadas durante o armazenamento de sementes de espécies florestais exóticas do Estado do Rio Grande do Sul. Santa Maria, RS, 2005

Table 1 -Year of collection, origin and number of analyses carried out during seed storage of exotic forest species of the Rio Grande do Sul. Santa Maria, RS - 2005

\begin{tabular}{|c|c|c|c|}
\hline Espécie & Ano de Coleta & Procedência/Lote & Número de Análises \\
\hline \multirow[t]{7}{*}{ Callistemon speciosus } & 1997 & Estação Experimental Santa Maria & 7 \\
\hline & 1997 & Santa Maria & 7 \\
\hline & 1997 & Boca do Monte & 7 \\
\hline & 1998 & Santa Maria & 8 \\
\hline & 1999 & Estação Experimental Santa Maria & 7 \\
\hline & 1997 & Santa Maria & 4 \\
\hline & 1996 & Veranópolis & 4 \\
\hline \multirow{2}{*}{ Cassia fistula } & 1998 & Restinga Seca & 9 \\
\hline & 1998 & Boca do Monte & 4 \\
\hline \multirow[t]{3}{*}{ Eucalyptus grandis } & 1998 & Santana do Livramento & 4 \\
\hline & 1998 & Santana do Livramento & 3 \\
\hline & 2001 & Santana do Livramento & 3 \\
\hline \multirow[t]{3}{*}{ Eucalyptus robusta } & 1995 & São Pedro do Sul & 6 \\
\hline & 1990 & Santo Augusto & 6 \\
\hline & 1999 & Santo Augusto & 6 \\
\hline \multirow[t]{3}{*}{ Acácia caven } & 1998 & Restinga Seca & 5 \\
\hline & 1999 & Restinga Seca & 3 \\
\hline & 1998 & Boca do Monte & 4 \\
\hline \multirow[t]{5}{*}{ Delonix regia } & 1993 & Santa Maria & 9 \\
\hline & 1995 & São Borja & 7 \\
\hline & 1997 & São Borja & 6 \\
\hline & 1998 & São Borja & 5 \\
\hline & 1999 & São Borja & 6 \\
\hline \multirow[t]{4}{*}{ Jacaranda mimosaefolia } & 1996 & Júlio de Castilhos & 6 \\
\hline & 1997 & São Borja & 7 \\
\hline & 1998 & Ijuí & 5 \\
\hline & 1998 & Ijuí & 5 \\
\hline \multirow[t]{4}{*}{ Pinus elliottii } & 1997 & Faxinal do Soturno & 6 \\
\hline & 1998 & Faxinal do Soturno & 5 \\
\hline & 1998 & Itaara & 7 \\
\hline & 2001 & Faxinal do Soturno & 6 \\
\hline
\end{tabular}

R. Árvore, Viçosa-MG, v.30, n.4, p.567-574, 2006 
A partir dessas informações, calculou-se a matriz de correlações entre todas as variáveis e realizou-se o diagnóstico de multicolinearidade, em que se mantiveram na análise as variáveis que proporcionaram multicolinearidade fraca, ou seja, o número de condições (NC) menor que 100, conforme recomendação de Cruz e Regazzi (1994). Em seguida, aplicou-se a análise de trilha entre as variáveis restantes, em que a porcentagem de plântulas normais foi considerada como dependente principal, sendo as demais, permanecentes após o diagnóstico da multicolinearidade, as independentes. Para realização das análises estatísticas utilizou-se o programa GENES (CRUZ, 2001), aplicando o teste " $t$ " a 5\% de probabilidade de erro, para testar significância dos coeficientes de correlação.

\section{RESULTADOS E DISCUSSÃO}

Não foi possível a obtenção dos coeficientes de trilha devido à presença apenas de correlação simples entre as variáveis umidade (U\%) e porcentagem de plântulas normais (PPN), para as espécies Calistemon specious, E. grandis e E.robusta com correlação de -0,3565, 0,1547 e 0,3809, respectivamente (Tabela 2). Esse fato resultou em coeficientes de determinação baixos $(0,127 ; 0,023$; e 0,145$)$ com os respectivos efeitos das variáveis residuais elevados $(0,93 ; 0,99$; e 0,92$)$, o que inviabilizou as análises estatísticas para as referidas espécies. Com isso, pode-se inferir que para aquelas espécies não há relações entre a percentagem de plântulas normais e as demais variáveis avaliadas nas análises de sementes, exceto entre U\% e PPN em $C$. specious, sendo essa inclusive significativa, mostrando que quanto menor a umidade da semente, maior será a germinação. Com essa comprovação, há como planejar o armazenamento de sementes de $C$. specious em umidades menores de conservação, fazendo que a germinação seja mantida em níveis ótimos para a espécie.

Outras correlações de interesse mostraram-se significativas, por exemplo a PUR x PMS para A. caven e J. mimosaefolia, cujo coeficiente-se mostrou-se negativo e, para P. elliottii, positivo (Tabela 3). O comportamento negativo indica que, para aumentar a pureza em um lote, há a necessidade de reduzir o peso de mil sementes desse, ou seja, no processo de preparo do lote devese dar maior atenção à pureza, com a eliminação adequada das "impurezas" existentes e tentar eliminar ao máximo as sementes que apresentam características de mortas e as maiores sementes. No caso do P. elliottii, em que, com o aumento no PMS, a pureza aumenta e o processo de composição de um lote fica mais facilitado, devendo dar preferência, nessa composição, às maiores sementes coletadas.

Tabela 2 - Coeficiente de correlação de Pearson entre as variáveis pureza (PUR) e peso de mil sementes (PMS), em gramas, e porcentagens de umidade (U\%), de germinação das plântulas normais (PPN), de sementes duras (PSD), de plântulas anormais (PPA) e de sementes mortas (PSM) em lotes de sementes de espécies exóticas do Rio Grande do Sul. Santa Maria, RS, 2005

Table 2 - Correlation coefficient among the variables purity $(P U R)$, thousand seed weight (PMS), seed moisture content (U\%), normal seedlings (PPN), hard seeds (PSD), abnormal seedlings (PPA) and dead seeds (PSM) in seed lots of exotic forest species of Rio Grande do Sul. Santa Maria-RS, 2005

\begin{tabular}{|c|c|c|c|c|c|}
\hline & & \multicolumn{3}{|c|}{ Espécies florestais } & \multirow[b]{2}{*}{ E.robusta } \\
\hline & & Delonix regia & Calistemon specious & E. grandis & \\
\hline \multirow[t]{6}{*}{$\overline{\text { PUR } x}$} & PMS & 0,2091 & - & - & - \\
\hline & $\mathrm{U} \%$ & 0,0088 & & & - \\
\hline & PPN & 0,2801 & & & 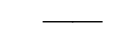 \\
\hline & PSD & $-0,1504$ & & & 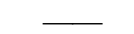 \\
\hline & PPA & 0,0296 & & 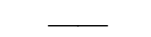 & 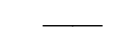 \\
\hline & PSM & $-0,2754$ & & 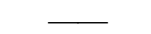 & 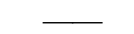 \\
\hline \multirow[t]{5}{*}{ PMS $\mathrm{x}$} & $\mathrm{U} \%$ & $-0,0985$ & 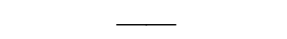 & 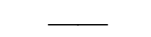 & 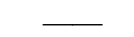 \\
\hline & PPN & 0,3395 & 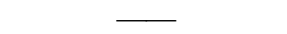 & 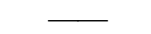 & 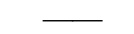 \\
\hline & PSD & $-0,1709$ & 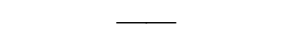 & . & 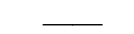 \\
\hline & PPA & $-0,0357$ & - & 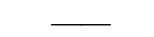 & 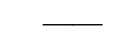 \\
\hline & PSM & $-0,341 *$ & 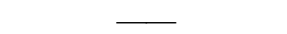 & 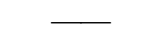 & 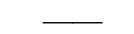 \\
\hline \multirow[t]{4}{*}{$\mathrm{U} \% \mathrm{x}$} & PPN & $-0,2232$ & $-0,3565 *$ & 0,1547 & 0,3809 \\
\hline & PSD & $-0,0171$ & 0,0000 & 0,0000 & 0,0000 \\
\hline & PPA & 0,0569 & 0,0000 & 0,0000 & 0,0000 \\
\hline & PSM & $0,3866 *$ & 0,0000 & 0,0000 & 0,0000 \\
\hline \multirow[t]{3}{*}{ PPN $x$} & PSD & $-0,7689 *$ & 0,0000 & 0,0000 & 0,0000 \\
\hline & PPA & $-0,3422 *$ & 0,0000 & 0,0000 & 0,0000 \\
\hline & PSM & $-0,7193 *$ & 0,0000 & 0,0000 & 0,0000 \\
\hline \multirow[t]{2}{*}{ PSD $x$} & PPA & 0,1886 & 0,0000 & 0,0000 & 0,0000 \\
\hline & PSM & 0,1092 & 0,0000 & 0,0000 & 0,0000 \\
\hline PPA $x$ & PSM & 0,3059 & 0,0000 & 0,0000 & 0,0000 \\
\hline
\end{tabular}

* significativo a $5 \%$ de probabilidade de erro, pelo teste "t".

R. Árvore, Viçosa-MG, v.30, n.4, p.567-574, 2006 
Tabela 3 - Coeficiente de correlação de Pearson entre as variáveis pureza (PUR) e peso de mil sementes (PMS), em gramas, e percentagens de umidade (U\%), de germinação das plântulas normais (PPN), de sementes duras (PSD), de plântulas anormais (PPA) e de sementes mortas (PSM) em lotes de sementes de espécies exóticas do Rio Grande do Sul. Santa Maria, RS, 2005

Table 3 - Correlation coefficient among the variables purity (PUR) thousand seed weight (PMS), seed moisture content (U\%), normal seedlings (PPN), hard seeds (PSD), abnormal seedlings (PPA) and dead seeds (PSM) in seed lots of exotic forest species of Rio Grande do Sul. Santa Maria - RS, 2005

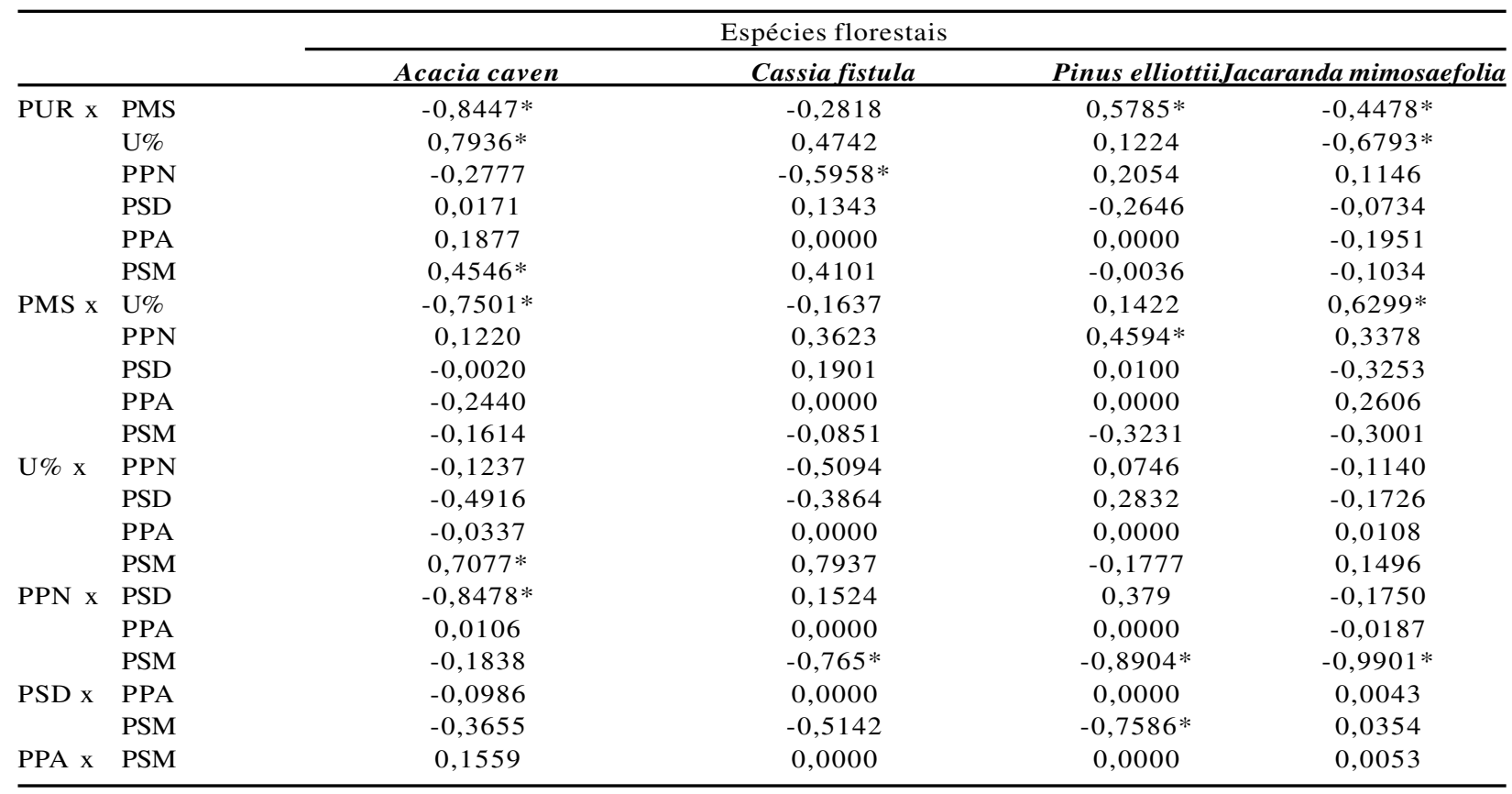

* significativo a $5 \%$ de probabilidade de erro, pelo teste " $\mathrm{t}$ ".

Nas relações da PPN com as demais variáveis, verificou-se (Tabelas 2 e 3 ) que em todas as espécies estudadas pelo menos um coeficiente de correlação mostrou-se significativo, alguns óbvios como PPN x PSM, PPN x PSD e PPN x PPA, apresentando coeficientes negativos. Uma correlação linear interessante nesse caso ocorreu entre PMS x PPN no P. elliottii, sendo positiva, evidenciando que nessa espécie, ao escolher as maiores sementes coletadas para comporem um lote, aumentam-se a pureza e também a porcentagem de plântulas normais, prática essa de certa facilidade a ser adotada no preparo de um lote a ser armazenado.

$\mathrm{Na}$ interpretação dos resultados obtidos nas decomposições do coeficiente de correlação linear de Pearson em efeitos direto e indireto (Tabela 4), devese considerar que, quando o coeficiente de correlação e o efeito direto forem iguais ou semelhantes, em magnitude e sinal, essa correlação direta explica a verdadeira associação entre as variáveis (VENCOVSKY e BARRIGA, 1992). Se o coeficiente de correlação de Pearson for positivo, mas o efeito direto for negativo ou desprezível, a correlação será causada pelos efeitos indiretos, sendo estes considerados na análise. Com o coeficiente de correlação de Pearson desprezível e o efeito direto, apresentando-se positivo e alto, os efeitos indiretos é que são responsáveis pela falta de correlação, merecendo a mesma atenção na análise. Finalmente, com a correlação de Pearson negativa e efeito direto positivo e alto, devem-se eliminar os efeitos indiretos da análise e aproveitar somente os diretos.

Em Acacia caven, apesar de o coeficiente de Pearson ter apresentado valor baixo e negativo entre U\% e PPN, seu efeito direto foi muito alto $(2,138)$, indicando que existe efeito indireto negativo e alto do PMS e PSM, e o PMS apresentou efeito direto alto, reduzido consideravelmente pelo efeito alto e negativo da umidade.

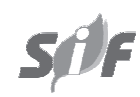

R. Árvore, Viçosa-MG, v.30, n.4, p.567-574, 2006 
Tabela 4 - Estimativas dos efeitos direto e indireto e do coeficiente de determinação $\left(\mathrm{R}^{2}\right)$ obtidas pela análise de trilha, entre as variáveis pureza (PUR) e peso de mil sementes (PMS), em gramas, e porcentagens de umidade (U\%), de plântulas anormais (PPA), de sementes duras (PSD) e de sementes mortas (PSM) sobre a porcentagem de germinação das plântulas normais (PPN), em lotes de espécies exóticas do Rio Grande do Sul. Santa Maria, RS, 2005

Table 4 - Path analysis for the variables purity (PUR), thousand seed weight (PMS), seed moisture content (U\%), hard seeds (PSD), abnormal seedlings (PPA) and dead seeds (PSM) on normal seedlings (PPN), in seed lots of exotic forest species of Rio Grande do Sul. Santa Maria-RS, 2005

\begin{tabular}{|c|c|c|c|c|c|c|c|}
\hline Efeitos & & PUR & PMS & U\% & PPA & PSD & PSM \\
\hline & & \multicolumn{6}{|c|}{ Acacia caven } \\
\hline Direto & & 0,199 & 1,787 & 2,138 & $-0,362$ & 0,717 & $-1,743$ \\
\hline Indireto via & PUR & - & $-0,168$ & 0,158 & 0,003 & 0,037 & 0,090 \\
\hline Indireto via & PMS & $-1,509$ & -- & $-1,340$ & $-0,003$ & $-0,436$ & $-0,288$ \\
\hline Indireto via & $\mathrm{U} \%$ & 1,697 & $-1,604$ & - & $-1,051$ & $-0,072$ & 1,513 \\
\hline Indireto via & PPA & $-0,006$ & 0,000 & 0,178 & -- & 0,035 & 0,132 \\
\hline Indireto via & PSD & 0,134 & $-0,175$ & $-0,024$ & $-0,070$ & - & 0,111 \\
\hline Indireto via & PSM & $-0,792$ & 0,281 & $-1,233$ & 0,637 & $-0,271$ & - \\
\hline \multirow[t]{2}{*}{$\mathrm{R}^{2}$} & 0.533 & \multirow{2}{*}{\multicolumn{6}{|c|}{ Cassia fistula }} \\
\hline & & & & & & & \\
\hline Direto & & $-0,181$ & 0,345 & 0,446 & $-0,324$ & - & $-1,182$ \\
\hline Indireto via & PUR & -- & 0,051 & $-0,086$ & $-0,024$ & - & $-0,074$ \\
\hline Indireto via & PMS & $-0,097$ & - & $-0,056$ & 0,066 & - & $-0,029$ \\
\hline Indireto via & $\mathrm{U} \%$ & 0,211 & $-0,073$ & -- & $-0,172$ & - & 0,354 \\
\hline Indireto via & PPA & $-0,043$ & $-0,061$ & 0,125 & - & - & 0,166 \\
\hline Indireto via & PSM & $-0,484$ & 0,100 & $-0,938$ & 0,607 & - & - \\
\hline \multirow[t]{2}{*}{$\mathrm{R}^{2}$} & 0,860 & & & & & & \\
\hline & & \multicolumn{6}{|c|}{ Delonix regia } \\
\hline Direto & & 0,089 & 0,105 & 0,037 & & $-0,151$ & $-0,627$ \\
\hline Indireto via & PUR & - & 0,018 & 0,000 & - & 0,002 & $-0,026$ \\
\hline Indireto via & PMS & 0,022 & - & $-0,010$ & -- & $-0,003$ & $-0,035$ \\
\hline Indireto via & $\mathrm{U} \%$ & 0,000 & $-0,003$ & -- & - & 0,002 & 0,014 \\
\hline Indireto via & PSD & $-0,003$ & 0,005 & $-0,008$ & -- & -- & $-0,046$ \\
\hline Indireto via & PSM & 0,172 & 0,213 & $-0,242$ & - & $-0,191$ & - \\
\hline \multirow[t]{2}{*}{$\mathrm{R}^{2}$} & 0,555 & & & & & & \\
\hline & & \multicolumn{6}{|c|}{ Jacaranda mimosaefolia } \\
\hline Direto & & 0,094 & 0,682 & $-0,479$ & -- & - & - \\
\hline Indireto via & PUR & - & $-0,042$ & $-0,064$ & - & - & - \\
\hline Indireto via & PMS & $-0,305$ & -- & 0,430 & - & - & -- \\
\hline Indireto via & $\mathrm{U} \%$ & 0,325 & $-0,302$ & -- & -- & - & -- \\
\hline \multirow[t]{2}{*}{$\mathrm{R}^{2}$} & 0,296 & & & & - & -- & -- \\
\hline & & \multicolumn{6}{|c|}{ Pinus elliottii } \\
\hline Direto & & 0,158 & 0,100 & $-0,115$ & - & - & $-0,877$ \\
\hline Indireto via & PUR & -- & 0,091 & 0,019 & -- & -- & 0,000 \\
\hline Indireto via & PMS & 0,058 & -- & 0,014 & -- & - & $-0,033$ \\
\hline Indireto via & $\mathrm{U} \%$ & $-0,014$ & $-0,016$ & -- & -- & - & 0,020 \\
\hline Indireto via & PSM & 0,003 & 0,283 & 0,156 & - & - & - \\
\hline $\mathrm{R}^{2}$ & 0,851 & & & & & & \\
\hline
\end{tabular}

Já na espécie Cassia fistula, observou-se correspondência tanto de sinal quanto de magnitude entre o PSM e PMS e a PPN. Por isso, ao se realizar a escolha de um lote de sementes dessa espécie, é fundamental manter nos níveis mais baixos possíveis de sementes mortas, enquanto se devem escolher sementes grandes e pesadas para garantir altas porcentagens de germinação. Quanto à umidade, o coeficiente de Pearson estimado foi de $-0,509$, indicando que para uma alta PPN são necessários lotes com baixa umidade. Entretanto, seu efeito direto foi alto e positivo, evidenciando-se efeito indireto expressivo e negativo via PSM. 
A germinação das sementes de Delonix regia foi favorecida pela baixa porcentagem de sementes duras e sementes mortas (correlação e efeito direto negativos e altos). Também nessa espécie, faz-se necessário formar lotes com alta pureza e com sementes de maior tamanho, obtendo, assim, respostas favoráveis à boa formação de mudas.

A germinação adequada do Jacaranda mimosaefolia depende, principalmente, do tamanho de suas sementes, dando preferência às maiores sementes e às mais pesadas, além de manter as sementes com baixa umidade devido ao efeito direto alto e negativo dessa variável. Essa mesma determinação faz que a associação entre a porcentagem de plântulas normais e PMS fique mais baixa que seu efeito direto.

A porcentagem de plântulas normais no Pinus elliottii apresenta-se dependente, principalmente, de uma baixa porcentagem de sementes mortas (efeito direto de mesmo sinal e magnitude que a correlação de Pearson), de lotes puros com sementes pesadas. Essa análise indicou indiferença da espécie à umidade das sementes, em que o efeito direto e negativo da $\mathrm{U} \%$ foi compensado pelo efeito indireto, de mesma magnitude e sinal contrários ao PMS.

Entre as espécies estudadas, houve concordância de que a porcentagem de sementes mortas é o principal fator que reduz a porcentagem de plântulas normais, como já era esperado, com exceção das sementes de Pinus, que se mostraram indiferentes a essa determinação. Nesse caso, a umidade foi a que apresentou relação inversa à PPN.

As relações da porcentagem de umidade (U\%) confirmam a importância dessa variável no armazenamento de sementes florestais. Hartmann e Kester (1974) consideraram que as condições efetivas para o armazenamento compreendem uma combinação de umidade relativa de 10 a $50 \%$ e uma temperatura de 0 a $10^{\circ} \mathrm{C}$. O teor de umidade das sementes é função da umidade relativa do ar ao seu redor e que, por sua vez, é influenciado pela temperatura de armazenamento. Em sementes de coníferas, um teor de umidade entre 5 e $8 \%$ tem-se mostrado efetivo (WANG, 1977). Os teores de umidade para conservação de sementes de diferentes espécies, pelo período de 1 ano, variam de aproximadamente 11 a 14\%, de acordo com Toledo e Marcos Filho (1977), verificando-se redução na porcentagem de plântulas normais à medida que aumenta o teor de umidade das sementes. Para o armazenamento por períodos mais longos, as sementes devem apresentar teores de umidade inferiores a $11 \%$, conforme Kano et al. (1978). Assim, estudos, das espécies avaliadas deverão ser conduzidos para o conhecimento do comportamento da germinação, em armazenamento das sementes em umidade relativa inferior a $10 \%$.

\section{CONCLUSÃO}

Para o aumento da porcentagem de plântulas normais, devem-se reduzir as porcentagens de sementes mortas e de umidade de armazenamento dos lotes de sementes das espécies florestais Acacia caven, Cassia fistula, Delonix regia, Jacaranda mimosaefolia e Pinus elliottii, armazenados nos Bancos de Sementes Florestais.

\section{AGRADECIMENTOS}

Ao Centro de Pesquisas Florestais e Conservação do Solo da Fundação Estadual de Pesquisa Agropecuária (FEPAGRO), pela disponibilidade dos dados para a realização do trabalho.

Ao CNPq e à FAPERGS, pelo auxílio financeiro.

\section{REFERÊNCIAS BIBLIOGRÁFICAS}

BRASIL. Ministério da Agricultura e Reforma Agrária. Secretaria Nacional de Defesa Agropecuária. Departamento Nacional de Defesa Vegetal. (1992). Regras para análise de sementes. Brasília: 1992. 365p

CRUZ, C.D. Programa GENES, versão windows: aplicativo computacional em genética e estatística. Viçosa, MG: Universidade Federal de Viçosa, 2001. 648p.

\section{CRUZ, C.D.; REGAZZI, A.J. Modelos \\ biométricos aplicados ao \\ melhoramento genético. Viçosa, MG:}

Universidade Federal de Viçosa, 1994. 390p.

GOEDERT, C.O.; SALOMÃO, A.N.; FAIAD, M.G. Germoplasma: o que é isso? Seednews, n.3, v.9, 2002. p. 16-21.

GUEDES, T.A. Introdução ao controle da qualidade. Melhoria contínua da qualidade. Semana da Estatística, 2., Curitiba: UEM/ DES, 2002. 58p.

R. Árvore, Viçosa-MG, v.30, n.4, p.567-574, 2006 
HARTMANN, H. T; KESTER, D. E. Propagacion de las plantas. México: Continental, 1974. 810p.

KANO, N.K.; MÁRQUEZ, F.C.M.; KAGEYAMA, P.Y. Armazenamento de sementes de ipê-dourado (Tabebuia sp). IPEF, n. 17, p. 13-23, 1978.

LÚCIO, A. D. Erro experimental relacionado às características dos ensaios nacionais de competição de cultivares. 1999. 73f. Tese (Doutorado em Agronomia), Faculdade de Ciências Agrárias e Veterinárias Campus de Jaboticabal, Jaboticabal, 1999.

MACHADO, C.F. Metodologia para a condução do teste de germinação e utilização de raios-X para a avaliação da qualidade de sementes de aroeirabranca (Lithraea molleoides (Vell.) Engl. 2002. 86f. Dissertação (Mestrado em Agronomia) Escola Superior de Agricultura Luiz de Queiroz, Piracicaba, 2002.
STORCK, L. et al. Experimentação vegetal. Santa Maria: Universidade Federal de Santa Maria, 2000. 198p.

TOLEDO, F. F.; MARCOS FILHO, J. Manual de sementes: tecnologia da produção. São Paulo: Agronômica Ceres, 1977. 224p.

VENCOVSKY, R.; BARRIGA, P. Genética biométrica no fitomelhoramento. Ribeirão Preto: Revista Brasileira de Genética, 1992. 496p.

VIEIRA R.D.; CARVALHO N.M. Teste de vigor em sementes. Jaboticabal: FUNEP, 1994. 164p.

WANG, B. S. P. Procurement, handling and storage of tree seed for genetic research. WORLD CONSULTA TION ON TREE BREEDING, 3., 1977. Proceedings... Canberra: 1977. 548p. 